\title{
Stuart Seide : présentations du Songe d'une nuit d'été
}

Mise en scène pour le théâtre de Chaillot, janvier 1982

\section{Stuart Seide}

Marie-Thérèse Jones-Davies (éd.)

\section{(2) OpenEdition \\ Journals}

\section{Édition électronique}

URL : http://journals.openedition.org/shakespeare/453

DOI : 10.4000/shakespeare.453

ISSN : 2271-6424

Éditeur

Société Française Shakespeare

Édition imprimée

Date de publication : 1 novembre 1981

Pagination : 247-250

ISBN : 2-86433-016-4

Référence électronique

Stuart Seide, "Stuart Seide : présentations du Songe d'une nuit d'été », Actes des congrès de la Société française Shakespeare [En ligne], 3 | 1981, mis en ligne le 01 novembre 2007, consulté le 22 janvier 2020. URL : http://journals.openedition.org/shakespeare/453 ; DOI : 10.4000/shakespeare.453 


\title{
THEATRE ET IDEOLOGIES :
} Marlowe, Shakespeare

\author{
DIRECTEUR DE LA PUBLICATION \\ M.T. Jones - Davies
}

JEAN TOUZOT Libraire - Editeur

38 , rue Saint-Sulpice 75278 PARIS CEDEX 061982 
1982 Jean Touzot Libiaire-Editeur, Paris.

Reproduction et traduction mzme partielle interdite.

Tous droits réservts potir lous pays

y compris i'U,R.S.S. et les pays scandinaves

ISBN 2-86433-016-4 


\title{
AVANT-PROPOS
}

L'opposition soulignée par Horace entre éthique et esthétique Aut prodesse volunt aut delectare poetae - que les Elisabéthains aiment à évoquer, pourrait être le lien qui rattache les unes aux autres les communications, rencontres et table ronde de notre troisième congrès (1981) sur le thème : "Théátre et Idéologies: Marlowe, Shakespeare.» Toutefois, le résultat de nos échanges montre un dépassement possible de l'alternative éthique / esthétique, puisqu'il met en valeur l'insertion d'idéologies susceptibles de transformer la fonction même de la littérature.

Les pièces analysées dans le contexte de leur temps révèlent de multiples aspects de la vision du monde et de la place que l'homme $y$ occupe, tels que les projette le théâtre anglais au tournant du XVIe et au début du XVIIe siècle.

Une exploration du langage et de la pensée des deux grands dramaturges aboutit à un commentaire sur les courants philosophiques ou religieux, sur les idées parfois contradictoires et sur la curiosité intellectuelle jamais assouvie qui se font jour à travers la rhétorique de la scène et le jeu dramatique, du Faust de Marlowe à La Tempéte de Shakespeare.

\author{
M.T. jones-Davies
}

Présidente de la Société Française Shakespeare 
SOCIETE FRANCAISE SHAKESPEARE Actes de Congrès.1981

\section{THEATRE ET IDEOLOGIES : Marlowe, Shakespeare}

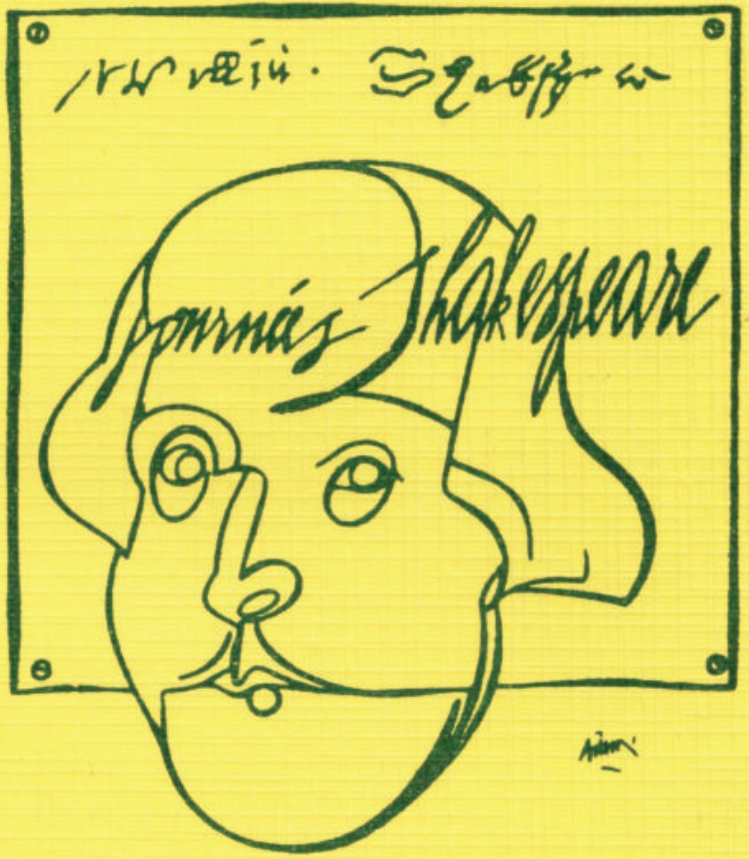

DIRECTEUR DE LA PUBLICATION M.T. Jones - Davies

JEAN TOUZOT Libraire - Editeur 38 , rue Saint-Sulpice 75278 PARIS CEDEX 061982 
Illustration de la couverture : affiche de Valerio Adami, spécialement conçue pour les Journées Shakespeare 1979 au Centre Georges Pompidou 


\section{TABLE DES MATIERES}

M.T. JONES-DAVIES Avant-propos

Théâtre et Idéologies: Marlowe, Shakespeare

S. TRUCHET

The Alchemy of Beauty: aesthetic principles in I Tamburlaine and their relationship to the hermetic ideology.

D. PRUDHOMME

L'idéologie du Juif de Malte et du Marchand de Venise.

D. GOY-BLANQUET

De Hall à Shakespeare : quelques glissements idéologiques opérés par la dramatisation dans Henry $I V$.

M.T. JONES-DAVIES Le Monıde du «si»: Idéologies Incertaines dans Comme il vous Plaira.

G. VENET

Temps et Idéologie : Marlowe, Shakespeare.

J. RICHER

Les Sept Caractères et les Quatre Tempéraments dans Jules César de Shakespeare.

F. LAROQUE

En marge de l'Idéologie : Antimasque et Grotesque dans le Dr Faustus et La Tempête.

A. LECERCLE-SWEET Conscience et Méconnaissance dans Edward The Second de Marlowe.

M.A. CONEJERO Shakespeare's Aesthe tic Scheme. (Extrait).

P.STEWART

Shylock, Shakespeare's Alien.

(Royal Shakespeare Company)

J.RUSSELL BROWN The Romantics' Shakespe are.

R. GILL

The Christian Ideology of Dr Faustus.

J. JACQUOT

Marlowe : de quelques problèmes d'interprétation. 
Rencontres entre Universitaires et Gens de Théâtre

Vendredi 4 décembre 1981
- Stuart Seide :Présentation du

Songe d'une nuit d'Eté. Mise en scène pour le Théâtre de Chaillot janvier 1982

- A propos d'Edouard (II) de

Bernard Turle

Bernard Turle : Métamorphoses de l'auteur et de l'écriture dramatiques. Intervention de Colin Harris (metteur en scène) 
RENCONTRES ENTRE UNIVERSITAIRES

ET GENS DE THEATRE 


\section{STUART SEIDE : PRESENT ATION DU SONGE D'UNE NUIT D'ETE \\ MISE EN SCENE POUR LE THEATRE DE CHAILLOT JANVIER 1982}

II y a dans le Songe plusieurs thèmes évidents, mais qui sont difficiles à mettre en évidence. D'abord, on se demande quelle est l'intrigue principale. Une fois que la pièce entre en répétition, la question ne se pose plus, ou elle se pose autrement.

L'intrigue consiste en une série d'accouplements, tous interrompus - sauf peut-être celui de Titania - y compris le premier, celuj de Thésée et Hyppoly te, qui se voit soumis à une attente de quatre jours. Tous les couples sont réunis dans le cinquième acte, qui est l'aboutissement de la pièce, alors que tous les fils de l'intrigue sont résolus dès l'acte IV. Et nous assistons à une représentation de Pyrame et Thisbé

Or Pyrame et Thisbé n'est rien d'autre qu'une version grotesque de Roméo et Juliette, pièce contemporaine du Songe. Il est fort probable que Shakespeare et sa compagnie jouaient les deux en alternance, la pièce sérieuse et la parodie. Cette histoire d'amour, est-elle tragique, ou est-elle ridicule? Pyrame et Thisbé, annoncent ses auteurs, est "une comédie du plus grand tragique»: on ne pourrait pas donner de meilleure définition du Songe. Et lorsque Thésée déclare, «je ne croirai jamais à ces fables» qu'inventent le fou, l'amant, le poète, on ne sait s'il parle de ce que les amants ont raconté, ou bien des quatre premiers actes de la pièce. Le texte joue ainsi constamment de ces renvois.

Le même système d'échos relie des intrigues apparemment indépendantes. Obéron et Titania sont en conflit, et c'est le cataclysme. Quand on est «out of love», quand on n'est pas en amour, il ne fait pas beau. Car aucun événement ne se produit isolément, tout ce qui se passe dans la voûte céleste a des résonances partout ailleurs, jusque dans les boutiques des artisans, dans les mondes apparemment séparés. Il y a des concordances entre le monde des mortels et celui des fées, qui toutes renvoient à l'amour, à la folie de l'amour.

Les artisans sont d'abord des amateurs de théâtre, rien d'autre, le reste est du folklore. Cela nous vaut le rare 
privilège d'assister à une répétition; même les acteurs de Hamlet ne font pas une véritable répétition. Tout ça pour jouer encore une histoire d'un amoureux et d'une amoureuse, cui se tuent, devant six amants. Parce que ces amateurs font apparaitre quelque chose qui avait échappé aux autres, l'histoire représentée ressemble à la leur, mais la seule personne dans toute cette pièce qui soit capable d'un véritable amour, non dominateur, l'amoureux le plus généreux, c'est le jeune homme qui tient le rôle de Thisbé et qui ne voulait même pas jouer le rôle, un amoureux de théâtre. On comprend alors pourquoi il faut du théâtre dans la cité.

Ce sont là quelques axes du travail de mise en scène. Le spectacle s'est organisé à partir de ces données, qui ont orienté nos choix. Par exemple, comment allions-nous traiter l'élément féérique de la pièce ? J'ai vu récemment une (mauvaise) mise en scène du Songe, et j'ai été gêné par la fleur magique. Tout dans la pièce peut être pris au pied de la lettre; mais ce qui est important, c'est la "changeance»*, la mouvance. Démétrius va rester envoûté à la fin de la pièce, il est le seul qui ne retrouve pas sa condition initiale, mais personne ne remarque la différence. Le philtre d'amour n'est qu'un catalyseur; la capacité d'inconstance devait être dans les personnages dès le début, la «changeance» était déjà là. Il y a toujours chez Shakespeare un fond de vérité psychologique étonnant sous l'apparence irrationnelle.

Il y aura donc peu de trucages. La magie est ailleurs, dans le texte même, les mots employés, pas tellement dans les excentricités vestimentaires, poudres et autres tapis volants. On veut que le monde soit différent, on le dit avec ferveur, et c'est cette ferveur qui fait l'incantation. Aucun accessoire ne peut être aussi magique que celui de notre imagination. On ne verra rien, pas de fleur magique, pas de «féerie».

Bernard Cavanna a composé une musique originale, avec quelques percussions. La vraie magie, c'est toujours la magie humaine, le dépassement. Un chanteur qui parvient à la pleine maitrise de sa voix, ou un sportif capable de franchir $2,60 \mathrm{~m}$ - eux réalisent la potentialité qui est en chacun de nous, et c'est ça qui est magique. Une bande enregistrée, ce n'est pas magique, c'est technologique.

La conception du décor est liée à ce que représente

- Terme utilisé par Stuart Seide 
la forêt dans le texte. La forêt du Songe, c'est le lieu où on se perd. Et où on se retrouve. Les amants fuient le monde des hommes et vont dans la nature pour être libres : or ils ne le sont pas, ils trouvent d'autres contraintes, puisqu'ils partent avec eux-mémes. Confrontés à la loi de la jungle, ils tinissent par se battre en duel. Une loi est remplacée par une autre mais peut-on dire que l'une est meilleure que l'autre ? Cette forét n'est pas un refuge paisible, elle est grouillante de choses maléfiques, au point que les fées font une carapace incantatoire autour de Titania pour protéger son sommeil. Plutôt qu'un lieu spécifique, la forêt est une absence, le manque du palais, le manque des structures de la cité; il y a opposition-complémentarité entre les lieux du drame. La forêt en tant que telle n'existe pas, elle est un chemin initiatique nécessaire.

Alors que met-on sur la scène quand on n'a pas vraiment besoin de décor ? En pleine rue, n'importe où, on pourrait se passer complètement de décor, mais c'est impossible dans un théâtre traditionnel, on ne peut pas jouer avec rien, dans un espace vide, car la neutralité n'existe pas. Notre décor sera noir, pour qu'on l'oublie le plus vite possible; des feuillages noirs, composant les murs d'un labyrinthe comme on en voit dans les jardins anglais. Et ce sera très éclairé; depuis la mise en scène de Peter Brook. on sait que le Songe, ça se joue en plein jour. Nous voulons apporter au spectateur juste assez de matière pour qu'il fasse le reste du chemin - si c'est trop peu, rien ne passe, et si c'est trop, on tue l'imagination - juste de quoi rèver son propre spectacle.

Enfin, plusieurs données sont intervenues dans le choix des costumes. Ceux des nobles s'inspirent du Premier Empire; parce qu'on ne peut pas oublier le Songe de Mendelssohn, ni Kleist et la Marquise d'O; et aussi parce qu'on retrouve l'évocation de la Grèce antique dans la mode du Premier Empire, avec un contenu érotique qui est une donnée importante de la pièce. Il ne s'agit pas de reconstituer l'époque, mais d'en présenter une épure.

Pour jouer Obéron et Titania, Thésée et Hippolyte ajoutent à leur costume un kimono, et une sorte de sari indien, qui donnent au spectacle sa référence orientale. fréquente dans la pièce. L'Inde du texte est un orient cérébral, l'autre côté du monde; ce monde imaginé est la 
face cachée du nôtre, l'autre côté de nous-mêmes. Quant aux artisans, ils seront en costume contemporain et ne porteront aucun signe de leur métier, car leur véritable artisanat, c'est le théâtre.

Le Songe est une pièce comico-tragico-politico-socioéconomique - et une pastorale; une pièce qui tient le miroir. Ils nous font rire, et ils nous font pleurer. A la fin de la représentation théâtrale, l'ensemble des cercles s'est refermé, les couples reconstitués, la construction est parfaite. Pourtant la pièce n'est pas terminée : c'est alors qu'Obéron et Titania entrent pour dire à tous «Au lit», et Puck conclut en rappelant au public qu'il n'est en fait qu'un pauvre acteur. Pourquoi cette entrée des fées une fois que tout est fini ? ça n'est pas nécessaire ... Donc c'est nécessaire : l'épisode ajoute un cercle supplémentaire à cette construction où chacun s'off re en spectacle bouffon à des spectateurs invisibles. Nous regardons les fées, qui regardent les nobles, qui regardent les artistes amateurs : le théatre dans le théâtre ...

Propos recueillis par Dominique Goy-Blanquet 4 décembre 1981 
A PROPOS D'EDOU/ARI) (II), de Bernard Turle, pièce écrite avec la collaboration dramaturgique de Colin Harris.

La soirée commence vers $21 \mathrm{~h} 00$, et se déroule en plusieurs étapes :

Présentation des invités : réunis autour de l'auteur Bernard Turle, et du metteur en scène Colin Harris, cinq comédiennes et comédiens, Agnès Ducios, Jean-Louis Heckel, Christine Régnier, Anna-Marie Vennel, et Didier Vuillecot.

Ensuite, deux interventions de la part de Bernard Turle et de Colin Harris, chacun parlant de sa participation au projet.

Bernard Turle : Métamorphoses de l'auteur et de l'écriture dramatiques.

Colin Harris : La modification du texte par le corps et autres dimensions de la représentation; la puissance du comédien.

Puis le groupe de sept personnes entame une lecture de courts extraits, destinés à mettre en lumière certains des thèmes de la pièce :

intimité du roi, son rapport avec Gaveston: tensions avec la reine Isabelle.

barons.

rapports de force, par exemple le conflit avec les

évolution de la situation dramatique : fuite d'Isabelle enceinte, tandis que les barons pourchassent Edouard et Gaveston: séparation du roi et de son ami; accouchement d'Isabelle.

retombées de la défaite de l'armée anglaise à Bannockburn; ascension de Lancastre.

Isabelle et Mortimer envahissent l'Angieterre; la répression sanglante; la fin de Despenser.

Faute de temps, le groupe a dû renoncer à l'étape prévue pour la fin de la soirée - un travail pratique commenté avec les comédiens à partir d'un des extraits qui viennent d'être lus.

La soirée s'est donc terminée par quelques remarques et appréciations sur la lecture de la part de l'assistance. Certaines comparaisons favorables - et pour les inter- 
venants flatteuses - ont été faites avec la pièce de T.S. Eliot Murder in the Cathedral.

Colin Harris, mars-avril 1982.

Annexes : Résumé de l'intervention de Bernard Turle et de Colin Harris. 


\section{PRESENTATION D'EDOUARD (II) \\ DE BERNARD TURLE collaboration dramaturgique de COLIN HARRIS}

Bernard Turle : Métamorphose de l'auteur et de l'écriture dramatiques.

A la source du spectacle, l'auteur est le membre de la troupe qui connaitra le plus de métamorphoses pendant le travail et la présentation du spectacle. Ceci ne se fait pas sans douleur. D'autant plus que c'est sa lente disparition du devant de la scène dont il est témoin. Pour conjurer cette souffrance, Colin Harris et moi avons décidé de travailler ensemble dès le départ, (sur un projet qui plaise à l'un et l'autre). L'écriture est donc dirigée vers la mise en scène et demeure presque illisible sans un appui visuel, etc ... que donne la représentation. L'écriture s'occupe principalement des sons, de la musique des mots, du ry thme, c'est.à _dire qu'on peut la taxer de lyrisme; on peut, à première vue, y trouver un texte non-théâtral. A mon avis, en effet, tout le théatre ne doit pas se trouver DEJA dans l'écrit. Colin Harris a d'ailleurs l'air de se trouver à l'aise dans ces mots qu'il aura toute liberté d'accompagner d'images. Les mots sont les miens, pourtant ils émanent de conversations que nous avons eues au fil des mois de gestation d'Edouard (II). Il peut donc s'y retrouver luimême sans avoir à plaquer une signification sur une oeuvre étrangère. Comme nous avons aussi parlé de mise en scène et modifié, voire supprimé des pans entiers de texte, comme des passages de batailles que nous n'imaginions pas du tout sur scène, en vue d'un ton général de mise en scène sinon d'une mise en scène en particulilier, je peux avoir l'espoir de me retrouver moi-même dans la future représentation et éviter ainsi les angoisses et colères de la dépossession dont je parlai plus haut et qui ne peuvent qu'accompagner un travail où l'acteur est le premier maillon de la chaine productrice, mais aussi très vite mis de cóté par une activité beaucoup plus extravertie que la sienne, celle des répétitions et des représentations.

EDOUARD (II) se démarque de ses prédecesseurs, les 
oeuvres de Marlowe et de Brecht. Sans doute Brecht est-il trop près de moi encore; je n'ai pas su le lire : je ne crois pas qu'on en entendra un écho ici. Je respecte beaucoup Marlowe et m'en sens même plus proche que de Shakespeare, mais il fallait aussi écarter Marlowe si l'on voulait, sans plaquer, une fois encore, une signification contemporaine sur une oeuvre du passé. Edouard (II) reprend donc la trame de la pièce de Marlowe. Mais le texte est entièrement nouveau, il est réécriture complète. En particulier, il était impossible d'avoir de l'histoire une approche qui se fonde à la vision élisabéthaine. Le moyen-âge est pour nous autant mythique que réaliste : les recherches nous en donnent une image plus serrée, pourtant notre imagination et le recul du temps (à travers les interprétations diverses : les pré-raphaëlites par exemple nous ont marqués) le lient au conte, à ses formes littéraires en général. Voilà donc une approche peu brechtienne du sujet. Car la distantiation est déjà dans la bouche des personnages (sont-ils d'ailleurs bien des personnages ? Ils sont d'abord des voix, expressions tronquées d'êtres dont nous ne percevons que des ombres, des reflets, mais certes pas un portrait total), elle se fait non seulement entre théatre et vie mais déjà entre vie et vie, celle qu'on mène et celle qu'on voudrait mener.

Autre glissement important du «mythe» d'Edouard, le rapport entre personnages est modifié dans la nouvelle version. J'ai peu de rapport avec la scène politique et je ne vois guère comment on peut la représenter au théátre sinon par une simplification encore plus outrée qu'elle n'est dans la vie courante. Les barons et les scènes qu'ils animent font donc pencher la pièce vers une bouffonnerie vulgaire et violente, un combat répétitif de marionnettes. S'il y a de la subtilité dans les tensions, il faut la chercher du côté des affects, des amants, des femmes, des amitiés. Edouard, oscillant entre les deux mondes, de par sa position reste un personnage prisonnier de son errance, sans message, fautif autant que victime. Peutêtre la force est-elle distribuée parmi les silencieux de la pièce plus que parmi les grands parleurs comme si la parole se retournait, tôt ou tard, contre ses utilisateurs. La prose, je l'espère, est forte, d'autant plus que les personnages sont, en fin de compte, faibles. Sa force ne réside pas dans un sens, quel qu'il soit. 
Je ne saurais donner aucune leçon. Je souhaite seulement qu'on entende le grondement d'une puissance non définie et peu exprimée mais seulement entenduc, perceptible dans le rythme, le llot qui entraine, porte ou broie les ètres. On pourrait facilement dire d'Edouard (II) que c'est un lieu (un temps) vide de sens. Et cela ne me gênerait pas. Parce que je le conçois, et Colin Harris est d'accord avec moi, bien davantage comme une oeuvre musicale ... Je ne veux que faire entendre - comme on peut écouter la mer, les remous et les vagues - les échos d'une histoire, légende ou mythe. De même que l'écoute d'un mythe est déjà une grande partie de sa compréhension, de méme Edouard (II) vise à faire comprendre par sa parole-même. là où l'exégèse tend souvent à se substituer au texte. 


\section{L'INTERVENTION de COLIN HARRIS : QUELQUES NOTES}

Le comédien a un potentiel de communication en dehors de tout texte; par sa seule présence, il peut créer une image ayant un sens précis. Le travail qu'il fait avec un instrument qui est son corps, dans toutes ses capacités expressives, peut aboutir à une modification du sens immédiat d'un texte donné.

Un point de départ pour la mise en scène : nous appuyer sur nos fantasmes par rapport à l'époque médiévale, les "projeter», pour ainsi dire, sur le passé. Donc, pas de tentative de "reconstituer» le Moyen Age, d'ailleurs mal connu. Nous ne savons pas précisément comment les gens vivaient le rapport avec leur propre corps. Ils avaient sûrement une conception très différente de la nôtre.

Ce corps que nous possédons est l'élément constant. Nous pouvons l'oublier, l'ignorer, même le maltraiter, mais nous ne pouvons nous en débarrasser sauf, bien súr solution extréme - en nous tuant ! Nous sommes bien obligés de "faire avec».

Par contre le langage, que nous avons tendance, grâce au mot écrit, à associer au fixe, au permanent, peut être considéré comme mobile, fluide, éphémère. Certes. il sert de raccourci, il est transmission de pensées et d'idées, échange et communication entre les êtres humains, mais discours ou dialogue - il reste néanmoins un «flot» qu'on ne retient pas.

$\mathrm{Au}$ théâtre, ces deux aspects, le corps matériellement présent de l'acteur et le flot du texte se trouvent réunis. Une réflexion sur la représentation théâtrale ne devait pas considérer l'un sans l'autre.

Bien que notre but avec Edouard (II) soit, parado$x a l e m e n t$, de privilégier le langage, faire entendre le texte. ceci ne pourra se faire sans la participation de l'acteur et son corps. Si le langage transmet un sens, le corps en véhicule un. lui aussi, le méme ou un autre, en principe parfaitement déchiffrable. Le corps est toujours "parlant». Il faut donc tenir compte de son "discours". Se limiter à proférer des paroles, sans se soucier du sens qui passe en même temps par son corps. c'est le mal qui frappe trop 
souvent le jeu du comédien français.

L'acteur est corps et langage à la fois. Un texte dit sans cette présence du corps ne s'étend pas. Mais méme en l'absence de langage, le corps "parle».Il "raconte» toujours quelque chose - ne serait-ce que sa propre maladresse, sa fatigue ou son inertie. A tout instant, il se donne comme évidence. Il ne peut pas "tricher», à moins qu'on ne le dissimule ... et encore ! Mais face à l'immense complexité de signes qu'il produit, on n'est guère capable de le «lire». Pourtant il s'offre toujours à une lecture. La tâche de l'acteur est donc de veiller à savoir ce que son corps raconte à chaque instant, en précisant au maximum les signes (images et gestes aussi).

Son travail corporel ou vocal peut à la limite changer entièrement le sens d'un texte. Puisqu'il fait vivre une image par son corps, il peut la jouer en contraste ou même en contradiction flagrante avec l'image suggérée par le texte. Ainsi peut naitre un nouveau sens, (dans le pire des cas un contresens), mais en tout cas, un troisième sens; et si le travail de création de l'image est entrepris consciemment. le rapport corps-texte peut donner lieu à un sens imprévu, étrange - sens parfois insoupçonné à travers la simple lecture d'une pièce.

Quelques notes supplémentaires :

l'écriture devait établir un lien direct avec le sujet. en contournant,pour ainsi dire, et Marlowe et Brecht.

- la structure dramatique de l'oeuvre, toute en séquences courtes, imposera un style de représentation : le montage (construction à partir du fragment).

l'espace scénique : un décor simplifié s'impose toiles, panoplie ou tenture - sinon, structures et accessoires réduits au strict minimum. Il faut faire confiance au jeu de l'acteur pour évoquer les espaces suggérés par l'écriture, ex: Cathédrale, lande, château ...

éléments de costumes : richesse des matières et des tissus (soie, laine, cuir, fourrure) et des couleurs (bleu, rouge, or). Parer le corps de l'acteur. Faire apparaitre le contraste : peau nue-habillement.

musique. Chant. Utiliser la musique pour créer un univers sonore qui dépayse le spectateur. Evocation de 
l'orient, qui, par son exotisme et son lien historique avec les croisades, reste source d'inspiration pour les arts, la mode, les légendes ...

le texte sera à tout instant contrainte, obligation. Par contre, l'acteur doit jouer (et jouir) librement de son corps comme un instrumentiste dans un ensemble de free jazz. Son travail portera à la fois sur l'écrit - le transformer en langue parlée ou chantée (travail vocal) - et sur le non-écrit - gestes, attitudes, regards, déplacements, etc. (travail corporel). 